\title{
A Missing Subepithelial Tumor in the Duodenum
}

\author{
Sam Ryong Jee \\ Department of Internal Medicine, Inje University College of Medicine, Busan, Korea
}

Question: A 43-year-old woman presented with a duodenal subepithelial tumor incidentally detected during screening endoscopy. Any specific symptoms and signs were not seen on the physical examination of the patient. There was no history of upper gastrointestinal bleeding or surgical interventions. The vital signs and laboratory findings were within the normal range. On esophagogastroduodenoscopy, a subepithelial mass was seen in the second portion of the duodenum, and its surface was covered by normal duodenal mucosa (Fig. 1A). The mass was so big that it almost obstructed the duodenal lumen. Abdominal CT showed duodeno-duodenal (first portion-second portion) intussusception (Fig. 1B, C), but the leading point of intussusception was not seen.

What is the most likely diagnosis?

Answer: A follow-up endoscopy was conducted. In the second portion of the duodenum, a large lumen ending in a blind pouch was observed (Fig. 2A). When the air was deflated, the blind pouch was pulled and shaped like a subepithelial tumor (Fig. 2B). A true lumen was seen above the blind pouch, and through the true lumen, the endoscope was entered to reach the third portion (Fig. 2C). Duodenogram revealed intraduodenal barium-filled sac with bulging of the thin radiolucent membrane in the second portion ("windsock" appearance) and incomplete obstruction with barium passage into the distal small bowel (Fig. 3).

On the basis of the findings described above, the patient was clinically diagnosed with intraluminal duodenal diverticulum (IDD). The duodenal diverticulum may be extraluminal or intraluminal. IDD is a congenital anomaly caused by an anomalous process of recanalization of the primitive foregut and is less common than extramural duodenal diverticulum. ${ }^{1}$
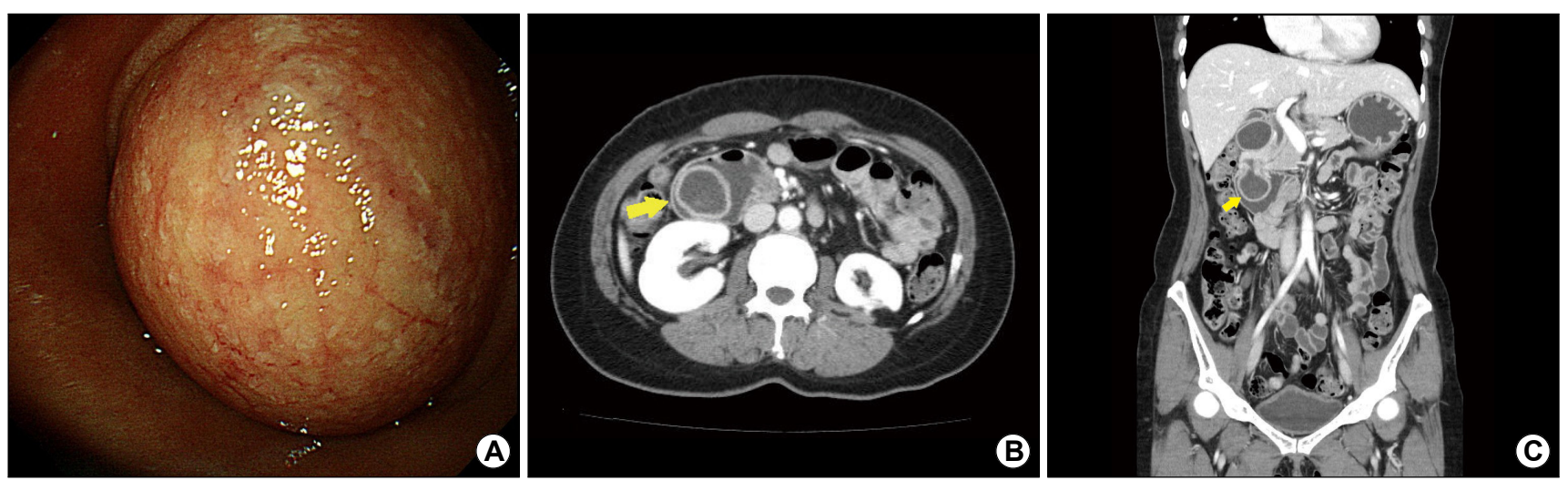

Fig. 1. (A) Endoscopy showing a subepithelial mass in the second portion of the duodenum, with its surface covered by normal duodenal mucosa. (B, C) Abdominal CT showing duodeno (first portion) - duodenal (second portion) intussusception (arrow). There is no visible leading point of the intussusception.

Received: July 13, 2020 Revised: July 22, 2020 Accepted: July 24, 2020

Corresponding author: Sam Ryong Jee

Department of Internal Medicine, Inje University College of Medicine, 75 Bokji-ro,

Busanjin-gu, Busan 47392, Korea

Tel: +82-51-890-6536, Fax: +82-51-892-0273, E-mail: srjee@inje.ac.kr

Copyright $\odot 2020$ Korean College of Helicobacter and Upper Gastrointestinal Research

(a) The Korean Journal of Helicobacter and Upper Gastrointestinal Research is an Open-Access Journal. All articles are distributed under the terms of the Creative Commons Attribution Non-Commercial License (http:// creativecommons.org/licenses/by-nc/4.0) which permits unrestricted non-commercial use, distribution, and reproduction in any medium, provided the original work is properly cited. 

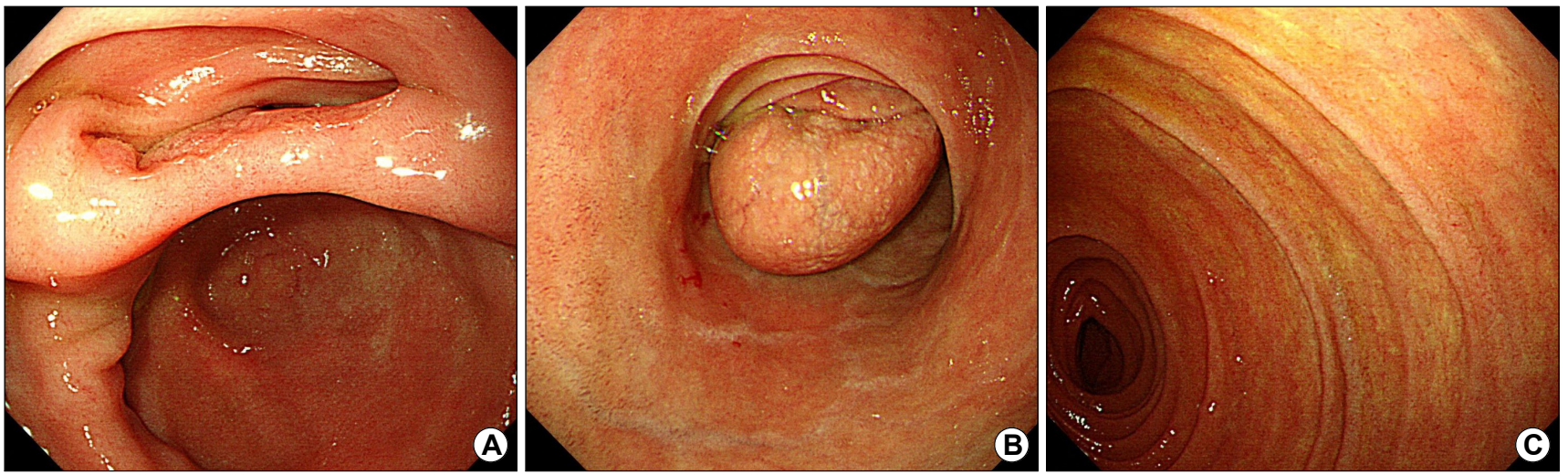

Fig. 2. (A) In the second portion of the duodenum, a large lumen ending in a blind pouch is observed. (B) When the air is deflated, the blind pouch is pulled and shaped like a subepithelial tumor. (C) A true lumen is seen above the blind pouch and through the lumen, the endoscope is inserted to reach the third portion.

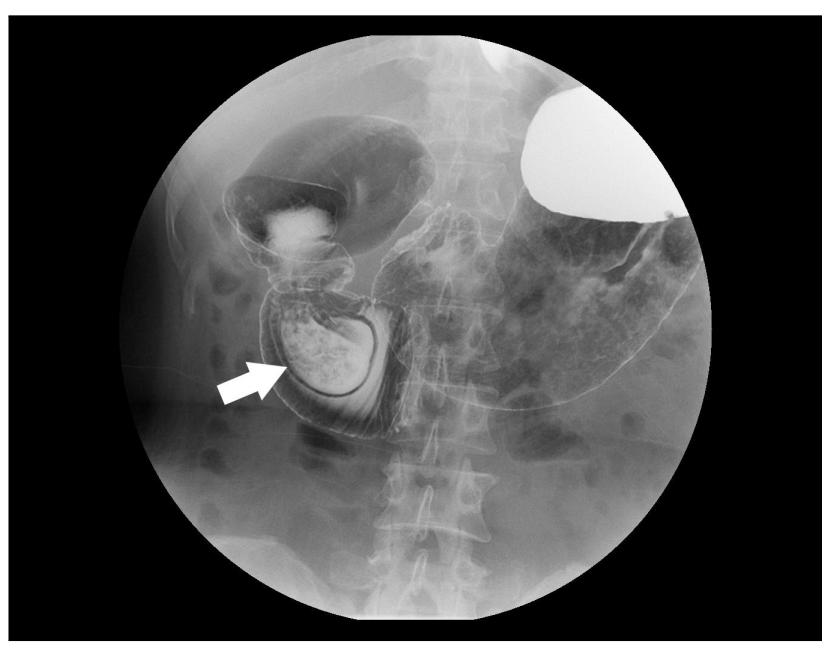

Fig. 3. Duodenogram revealing an intraduodenal barium-filled sac with bulging of the thin radiolucent membrane in the second portion ("windsock" appearance) (arrow) and incomplete obstruction with barium passage into the distal small bowel.

During childhood, significant obstructive symptoms may not occur due to an incomplete mucosal septum containing an aperture or fenestration. The duodenal diaphragm will gradually transform into a pulsion-type intraluminal diverticulum as force of peristalsis and duodenal content stretch the lumen continuously. IDD is diagnosed in both sexes between the third and fifth decade of life and is frequently associated with other abnormalities, such as annular pancreas, intestinal malrotation, and duplication of the papilla of Vater. ${ }^{2}$ The clinical presentation of IDD is rather nonspecific and may include nausea, epigastric pain, and vomiting. Acute pancreatitis and gastrointestinal bleeding also reported as possi- ble clinical manifestations.

The lesion is typically piriform, presenting with a proximal entrance orifice and a distal blind pouch. The classic appearance of the duodenogram is a pear-shaped pouch resembling a barium-filled "windsock", partially obstructing the duodenal lumen. ${ }^{3}$ The endoscopic findings are also characteristic but may be confusing if the examiner is not familiar with this rare lesion. The present case misled the interpretation as a subepithelial tumor in endoscopic diagnosis. In most cases, the IDD is connected to the duodenal wall posteriorly and projects intraluminally as a pocket. The passage of duodenal content is then through an opening located either centrally at the apex of the sac or somewhat eccentrically along one wall of the pouch. A slit-like aperture (true lumen) allows the endoscopy to be advanced distally for evaluation of the duodenal mucosa beyond the IDD.

The surgical method of choice is duodenotomy and excision of the intraluminal pouch. ${ }^{4}$ The endoscopic treatment with excision by snare cautery has been reported. ${ }^{5}$

\section{CONFLICT OF INTEREST}

No potential conflict of interest relevant to this article was reported.

\section{ORCID}

Sam Ryong Jee (D) https://orcid.org/0000-0002-7928-1153 


\section{REFERENCES}

1. Coors GA, Mitchum WR. Intraluminal duodenal diverticulum. Am J Surg 1962;103:400-402.

2. Takamatsu S, Gabata T, Matsui O, Noto M, Ninomiya I, Nonomura A. Intraluminal duodenal diverticulum: MR findings. Abdom Imaging 2006;31:39-42.

3. Fleming CR, Newcomer AD, Stephens DH, Carlson HC.
Intraluminal duodenal diverticulum. Report of two cases and review of the literature. Mayo Clin Proc 1975;50:244-248.

4. Economides NG, McBurney RP, Hamilton FH 3rd. Intraluminal duodenal diverticulum in the adult. Ann Surg 1977;185: 147-152.

5. Hajiro K, Yamamoto H, Matsui H, Yamamoto T. Endoscopic diagnosis and excision of intraluminal duodenal diverticulum. Gastrointest Endosc 1979;25:151-154. 